Article

\title{
New Positive Solutions of Nonlinear Elliptic PDEs
}

\author{
Mustafa Inc ${ }^{1}\left(\right.$, Noureddine Bouteraa ${ }^{2} \oplus$, Mehmet Ali Akinlar ${ }^{3}{ }^{\oplus}$, Slimane Benaicha ${ }^{2}$, \\ Yu-Ming Chu ${ }^{4,5, *(\mathbb{D})}$, Gerhard-Wilhelm Weber ${ }^{6,7}$ (D) and Bandar Almohsen ${ }^{8}$ \\ 1 Department of Mathematics, Firat University, Elazig 23119, Turkey; minc@firat.edu.tr \\ 2 Laboratory of Fundamental and Applied Mathematics of Oran (LMFAO), University of Oran1, \\ Ahmed Benbella 31000, Algeria; bouteraa-27@hotmail.fr (N.B.); slimanebenaicha@yahoo.fr (S.B.) \\ 3 Department of Mathematical Engineering, Yildiz Technical University, Istanbul 34220, Turkey; \\ mehmetaliakinlar@gmail.com \\ 4 Department of Mathematics, Huzhou University, Huzhou 313000, China \\ 5 Hunan Provincial Key Laboratory of Mathematical Modeling and Analysis in Engineering, \\ Changsha University of Science \& Technology, Changsha 410114, China \\ 6 Institute of Applied Mathematics, Poznan University of Technology, 61-138 Poznan, Poland; \\ gerhard.weber@put.poznan.pl \\ 7 IAM (UME), METU, Ankara 06800, Turkey \\ 8 Department of Mathematics, College of Science, King Saud University, P.O. Box 2455, Riyadh 11451, \\ Saudi Arabia; balmohsen@ksu.edu.sa \\ * Correspondence: chuyuming@zjhu.edu.cn
}

Received: 21 June 2020; Accepted: 13 July 2020; Published: 15 July 2020

\begin{abstract}
We are concerned with positive solutions of two types of nonlinear elliptic boundary value problems (BVPs). We present conditions for existence, uniqueness and multiple positive solutions of a first type of elliptic BVPs. For a second type of elliptic BVPs, we obtain conditions for existence and uniqueness of positive global solutions. We employ mathematical tools including strictly upper (SU) and strictly lower (SL) solutions, iterative sequence method and Amann theorem. We present our research findings in new original theorems. Finally, we summarize and indicate areas of future study and possible applications of the research work.
\end{abstract}

Keywords: positive (global) solution; (strict) upper and lower solutions; multiplicity of positive solutions; elliptic BVPs

\section{Introduction}

Nonlinear elliptic boundary value problems (NEBVP) are significantly important type PDEs having applications in different branches of science and engineering including fluid mechanics such as exothermic chemical reactions or auto catalytic reactions, see [1], in physics and chemistry. Some other specific applications of elliptic BVPs may be seen in [2-22].

The interest of such problems come from the thesis [23] (Section 1.5, page 147), where the authors asked open problem concerning multiplicity results. The main question we would like to address in this direction is the existence of more than two solutions, the articles of Chipot and Lovat [1] and Ovono and Rougirel [24], where the authors study classes of nonlocal problems motivated by the fact that they appear in some applied mathematics areas and the diffusion at each point depends on all the values of the solutions in a neighborhood of this point. Moreover, in [8], the authors have mentioned that the importance of such a model lies in the fact that measurements that serve to determine physical constants are not made at a point but represent an average in a neighborhood of a point so that these physical constants depend on local averages. The lack of the existence of the multiple solutions by using bifurcation theory showed that many local branches of solutions exist while, among them, 
only one is global and has no bifurcation point implying a considerable difficulty to prove the existence of a bifurcation point interior of the ball. The authors in [24] (cf. Theorem 3.1) already pointed out that the existence of a solution to the problem proposed (more exactly, for different kinds of NEBVP involving different conditions) is not guaranteed for the unboundedness of data $c$. It is natural to ask whether or not we can obtain the existence results of the EBVP or what happens if the data $c$ are unbounded. Up to now, the main scope of these papers consists in the imposing some conditions on the nonlinearity $c$ ( $c$ is the data) to prove the existence solutions to the problem (1) in smooth domains in the presence of well-ordered lower and upper solutions. Note that the case where $c$ is unboundedness seems to be new in the literature. In other words, we obtain the existence results under regularity assumptions on $c$, (see [25-28] for more discussion).

In the present research study, we are interested in existence, uniqueness, multiple positive solutions and existence of weak positive solutions for an NEBVP, (called first type NEBVP), defined as

$$
\begin{cases}-\mathcal{A} u=c(y, u), & \text { in } \Omega, \\ \mathcal{N} u=h(y), & \text { on } \partial \Omega,\end{cases}
$$

and positive global solutions for the second NEBVP defined as

$$
\begin{cases}u_{t}-\Delta u=c(y, t, u) & \text { in } \Theta, \\ \mathcal{N} u=h(y, t), & \text { on } \partial \Theta, \\ u(y, 0)=u_{0}(y), & \text { in } \Omega,\end{cases}
$$

in which $\Theta=\Omega \times(0, T], \partial \Theta=\partial \Omega \times(0, T], \Omega$ is a bounded set $\mathbb{R}^{n}$ with smooth boundary $\partial \Omega$, $\Delta u=\mathcal{A} u$ is a second order uniformly elliptic operator and $\mathcal{N}$ is defined as either

$$
\mathcal{N} u=u \text { on } \partial \Theta,
$$

or

$$
\mathcal{N} u=\lambda u_{v}+\mu u \quad \text { on } \partial \Theta,
$$

where $u_{v}$ is outward derivative in $\Omega, \lambda=\lambda(y, t), \mu=\mu(y, t)$ are bounded and strictly nonnegative maps on $\partial \Theta$. The initial non-negative smooth map $u_{0}(y)$ satisfies compatibility condition $u_{0}(y)=0$ on $\partial \Omega$.

The structure of the article is as follows. In Sections 2 and 3, we prove the existence, uniqueness and multiplicity of positive solutions for the first type and global positive solutions for the second type by employing strictly upper (SU) and strictly lower (SL) solutions, by iterative sequence method for both of them and the Amann theorem for the first type. Section 4 is devoted to the existence of a weak solution to the first type.

\section{Multiple Positive Solutions of Nonlinear Elliptic PDEs}

Definition 1. A function $\alpha \in C^{2}(\Omega) \cap C(\bar{\Omega})$ is said to be an upper solution (US) of (1) if $\alpha$ satisfies the following inequalities:

$$
\begin{cases}-\mathcal{A} \alpha \geq c(y, \alpha) & \text { in } \Omega, \\ \mathcal{N} \alpha \geq h, & \text { on } \partial \Omega .\end{cases}
$$

Moreover, a function $\beta \in C^{2}(\Omega) \cap C(\bar{\Omega})$ is a lower solution (LS) of (1) if for $\beta$ the conditions

$$
\begin{cases}-\mathcal{A} \beta \leq f(y, \beta) & \text { in } \Omega, \\ \mathcal{N} \beta \leq h, & \text { on } \partial \Omega,\end{cases}
$$

hold true. 
We say that a map $\underline{u}$ is a strict LS of (1) if,

(i) $\underline{u}$ is an LS of (1), and

(ii) $u>\underline{u}$ for all solutions of (1), such that $u(t) \geq \underline{u}(t)$ holds for all $t \in[0, T]$.

Similarly, a map $\bar{u}$ is an SU solution of (1) if:

(i) $\bar{u}$ is a US of (1) and

(ii) $u<\bar{u}$ for all solutions of (1) with $u(t) \leq \bar{u}(t)$ hold for all $t \in[0, T]$.

Now, we assume that there exist $\alpha, \beta \in C^{2}(\Omega) \cap C(\bar{\Omega})$ to (1), and define

$$
(\beta, \alpha)=\{u \in C(\bar{\Omega}): \beta \leq u \leq \alpha\} .
$$

$\left(H_{1}\right)$ Let $c$ be monotone nondecreasing in $u$ and $h>0$ such that

(i) for all $u_{i} \in \mathbb{R},(i=1,2)$ and for $y \in \Omega, c$ satisfies

$$
u_{1}<u_{2} \Rightarrow c\left(y, u_{1}\right)<c\left(y, u_{2}\right) .
$$

(ii) for $\alpha, \beta \in C^{2}(\Omega) \cap C(\bar{\Omega})$ with $\beta \leq \alpha$ on $\bar{\Theta}$, and

$$
\min \beta \leq u_{1} \leq u_{2} \leq \max \alpha,
$$

suppose that there exists $\sigma>0$ such that the inequality

$$
c\left(y, u_{1}\right)-c\left(y, u_{2}\right)>-\sigma\left(u_{1}-u_{2}\right) \text { holds. }
$$

(iii) for every $y \in \Omega, y^{\prime} \in \partial \Omega$, the inequalities $c(y, 0) \geq 0, h\left(y^{\prime}, 0\right) \geq 0, u_{0}(y) \geq 0$ hold.

We first make some observations on SU and SL solutions. We shall use the SU and SL solutions together with strong maximum principle in the sequel.

Lemma 1. (Strong maximum principle, see [29]) Let $\mathcal{F}, \mathcal{N}$, two elliptic operators, $\Omega$ be as in Section 1 and $v \in W^{2, p}(\Omega)$ be given. Then, the following holds.

(i) (Interior form) Let $y_{0} \in \Omega$ and let $B_{y_{0}}$ be an open ball centered at $y_{0}$ and contained $\Omega$. If $\mathcal{F} v \geq 0$ in $B_{y_{0}}$, $v(x) \geq v\left(y_{0}\right)$ for all $y \in B_{y_{0}}$ and $v\left(y_{0}\right) \leq 0$, then $v(y)=v\left(y_{0}\right)$ for all $y \in B_{y_{0}}$.

(ii) (Boundary form) Let $y_{0} \in \partial \Omega$ and let $B_{y_{0}}$ be an open ball contained in $\Omega$ with $y_{0} \in \partial B_{y_{0}}$. If $\mathcal{F} v \geq 0$ in $B_{y_{0}}, v(y) \geq v\left(y_{0}\right)$ for all $y \in B_{y_{0}}$ and $v\left(y_{0}\right) \leq 0$, then $\frac{\partial v}{\partial \zeta}\left(y_{0}\right)<0$ for each $\zeta$ satisfying $\langle\zeta \mid v\rangle>0$.

(iii) (Global form) Let $k \geq 0$ be a constant. If $\mathcal{F} v+k v \geq 0$ in $\Omega$ and $\mathcal{N} v \geq 0$ on $\partial \Omega$, then either $v=0$ in $\Omega$ or $v(y)>0$ for all $y \in \Omega \cup \partial \Theta$, and $\frac{\partial v}{\partial v}(y)<0$ for all $y \in \partial \Theta$, where $\mathcal{N}$ is defined as in Section 1.

Lemma 2. Let (5) hold and $u$ be any solution of (1). Then, every lower (upper, respectively) solution $\underline{u}(\bar{u})$, which is not a solution, (i.e., $u>\underline{u}(u<\bar{u})$ ) is an SL (SU) solution of (1).

Proof. Assume that $\underline{u}$ is an SL solution under hypotheses of Lemma 2. Let us prove this with a contradiction. Let $u, \underline{u}$ be any solutions with $u \geq \underline{u}$. Then,

$$
\begin{cases}-\mathcal{A}(u)=c(y, u), & \text { in } \Omega, \\ \mathcal{N}(u)=h(y), & \text { on } \partial \Omega .\end{cases}
$$

and

$$
\begin{cases}-\mathcal{A}(\underline{u}) \leq c(y, \underline{u}), & \text { in } \Omega, \\ \mathcal{N}(\underline{u}) \leq h(y), & \text { on } \partial \Omega .\end{cases}
$$


Putting $v=u-\underline{u}$ implies that $v>0$. By $u \geq \underline{u}$, and by the definition of the operator $\mathcal{A}$, a subtraction of above equations gives

$$
\begin{aligned}
-\mathcal{A} v & \leq-\mathcal{A} u+\mathcal{A} \underline{u} \quad \text { in } \Omega \\
& \leq c(y, u)-c(y, \underline{u}) .
\end{aligned}
$$

Thus, by hypothesis of $u=\underline{u}$ and (6), we have

$$
-\mathcal{A} v>-\sigma(u-\underline{u})=0,
$$

which is a contradiction that completes the proof.

We can prove that $\bar{u}$ is a strict US under given conditions in a similar manner.

Lemma 3. Assume that $\left(H_{1}\right)$ holds. Then, by defining $u, \bar{u}, \underline{u}$ as above, the inequalities

$$
\underline{u} \leq u \leq \bar{u},
$$

hold true.

Now, we are in a position to present some results regarding existence of solutions. To achieve this, starting from suitable maps $u^{(0)}=\alpha$ or $u^{(0)}=\beta$, obtain a sequence $\left\{u^{(n)}\right\}$ from

$$
\left\{\begin{array}{lr}
-(\mathcal{A}-\sigma) u^{(n)}=c\left(y, u^{(n-1)}\right)+\sigma u^{(n-1)}, & \text { in } \Omega, \\
\mathcal{N} u^{(n)}=h, & \text { on } \partial \Omega .
\end{array}\right.
$$

Lemma 4. Let $\alpha, \beta$, g be nonnegative, bounded functions and $\varphi \in C^{2}(\Omega)$ satisfying

$$
\begin{cases}-\mathcal{A} \varphi+g \varphi \geq 0, & \text { in } \Omega, \\ \mathcal{N} \varphi \geq 0, & \text { on } \partial \Omega .\end{cases}
$$

In this case, $\varphi \geq 0$ in $\bar{\Omega}$. Furthermore, $\varphi>0$ in $\Omega$, unless $\varphi=0$.

Theorem 1. Assume $\left(H_{1}\right)$ holds. Let $\alpha, \beta$ be LS and US of $(1)$ with $\beta \leq \alpha$ and $c(y, u)$ be a smooth map on $\min \beta \leq u \leq \max \alpha$. Then, there are two non-negative solutions $\bar{u}$ and $\underline{u}$ of problem (1) with

$$
\beta \leq \underline{u} \leq \bar{u} \leq \alpha .
$$

Proof. Obviously, by the hypothesis $\left(H_{1}\right)$ and $\beta \leq \alpha, \beta=0$ is an LS of (1).

Now, define $\mathcal{Q}: C(\bar{\Omega}) \mapsto C^{2}(\Omega) \cap C(\bar{\Omega})$ as $w=\mathcal{Q} u$, and

$$
\left\{\begin{array}{lr}
-(\mathcal{A}-\sigma) w=c(y, u)+\sigma u, & \text { in } \Omega \\
\mathcal{N} w=h, & \text { on } \partial \Omega .
\end{array}\right.
$$

By Schauder estimates, we deduce $\mathcal{Q}$ is completely continuous and monotonic in the sense of Collatz [30] type, that is; $u_{1} \leq u_{2}$ implies $\mathcal{Q} u_{1}<\mathcal{Q} u_{2}$, provided that $u_{1}$ and $u_{2}$ restricted to the set $\min \beta \leq u_{1}, u_{2} \leq \max \alpha$. In fact, if $u_{1} \leq u_{2}$, then

$$
\left\{\begin{array}{lr}
-(\mathcal{A}-\sigma)\left(\mathcal{Q} u_{2}-\mathcal{Q} u_{1}\right)=c\left(y, u_{2}\right)-c\left(y, u_{1}\right)+\sigma\left(u_{2}-u_{1}\right), & \text { in } \Omega, \\
\mathcal{N}\left(\mathcal{Q} u_{2}-\mathcal{Q} u_{1}\right)=0, & \text { on } \partial \Omega,
\end{array}\right.
$$


Then,

$$
\left\{\begin{array}{lr}
-\mathcal{A}\left(\mathcal{Q} u_{2}-\mathcal{Q} u_{1}\right) \geq-\sigma\left(u_{2}-u_{1}\right)+\sigma\left(u_{2}-u_{1}\right), & \text { in } \Omega, \\
\mathcal{N}\left(\mathcal{Q} u_{2}-\mathcal{Q} u_{1}\right)=0, & \text { on } \partial \Omega
\end{array}\right.
$$

i.e.,

$$
\left\{\begin{array}{lr}
-\mathcal{A}\left(\mathcal{Q} u_{2}-\mathcal{Q} u_{1}\right) \geq 0, & \text { in } \Omega, \\
\mathcal{N}\left(\mathcal{Q} u_{2}-\mathcal{Q} u_{1}\right)=0, & \text { on } \partial \Omega,
\end{array}\right.
$$

Hence, $\mathcal{Q} u_{1}<\mathcal{Q} u_{2}$, in $\Omega$.

$$
\begin{aligned}
& \text { Letting } u^{(0)}=\alpha \text { or } u^{(0)}=\beta \text {, generate }\left\{u^{(n)}\right\}=\left\{\mathcal{Q} u^{(n-1)}\right\} \text { as } \\
& \left\{\begin{array}{lr}
-(\mathcal{A}-\sigma) u^{(n)}=c\left(y, u^{(n-1)}(y)\right)+\sigma u^{(n-1)} \text { in } \Omega \\
\mathcal{N} u^{(k)}=h, & \text { on } \partial \Omega .
\end{array}\right.
\end{aligned}
$$

When $u^{(0)}=\alpha$, we set $\left\{\bar{u}^{(n)}\right\}$ and $\left\{\underline{u}^{(n)}\right\}$ when $u^{(0)}=\beta$. Then, the sequence $\left\{\bar{u}^{(n)}\right\}$ and $\left\{\underline{u}^{(n)}\right\}$ converges monotonically by the continuity of $\mathcal{Q}$ to $\bar{u}_{\max }$ and $\underline{u}_{\min }$, respectively. Thus, $\bar{u}=\bar{u}_{\max }$ and $\underline{u}=\underline{u}_{\min }$ are two fixed point of $\mathcal{Q}$. The proof is completed.

Corollary 1. Let $\left\{\bar{u}_{\max }\right\}$ and $\left\{\underline{u}_{\min }\right\}$ be two solutions of (1). If $w$ is a solution of (1) satisfying $\beta \leq w \leq \alpha$, the inequalities $\underline{u}_{\min } \leq w \leq \bar{u}_{\max }$ hold.

Proof. By Theorem 1, we have $w=\mathcal{Q} w$ and $\bar{u}_{1}=\mathcal{Q} \alpha$, since $w \leq \alpha, \mathcal{Q} w<\mathcal{Q} \alpha$ or $w<\bar{u}_{1}$.

By induction, $w \leq \bar{u}^{(n)}$ for every $n$. Thus, $w \leq \bar{u}_{\max }$. Similarly, $w \geq \underline{u}_{\min }$, hence $\underline{u}_{\min } \leq w \leq \bar{u}_{\max }$.

Theorem 2. Assume $\left(H_{1}\right)$ holds. (1) has positive local solution $u^{+}(y)$.

Proof. Notice that $\left(H_{1}\right)$ implies existence of LS and US. Then, by Theorem 1, there is a local positive solution $u^{+}(y)$ of $(1)$.

We adopt the following assumption:

$\left(H_{2}\right)$ Let $u_{1}, u_{2} \in(\beta, \alpha)$ with $u_{1} \leq u_{2}, c_{1}(y) \in \Omega$ be bounded nonnegative maps and the map $c(y, u)$ satisfies the following inequality

$$
c\left(y, u_{1}\right)-c\left(y, u_{2}\right) \geq-c_{1}(y)\left(u_{1}-u_{2}\right) \text { in } \Omega .
$$

Theorem 3. Let (5) and (iii) in $\left(H_{1}\right),\left(H_{2}\right)$ hold true. Assume also that $\beta(y), \alpha(y)$ are, LS and US of problem (1). Then, problem (1) has unique positive solutions in $(\beta, \alpha)$.

Proof. Existence of positive solutions of (1) may be observed by Theorem 1. Let $u_{1}, u_{2} \in(\beta, \alpha)$ be two poitive solutions with $u_{1} \leq u_{2}$. Suppose $w=u_{1}-u_{2}$, then $w \leq 0$ and by $\left(H_{2}\right)$, we have

$$
\left\{\begin{array}{l}
-A w=c\left(y, u_{1}\right)-c\left(y, u_{2}\right) \geq 0, \text { in } \Omega, \\
N w=h(y)-h(y)=0, \quad \text { on } \partial \Omega,
\end{array}\right.
$$

Applying Lemma 4 we have $u_{1}=u_{2}$.

By employing Amann Theorem [31], we show multiple positive solutions. Let $\alpha_{1}, \alpha_{2}$ are two upper solutions and $\beta_{1}, \beta_{2}$ are two lower solutions of problem (1). 
Theorem 4. ([31]) Assume that $E$ is a Banach space. Assume also that $K \subset E$ is a normal solid cone. Suppose that there are $\alpha_{1}, \alpha_{2}, \beta_{1}, \beta_{2} \in E$ by $\beta_{1}<\alpha_{1}<\beta_{2}<\alpha_{2}$. The operator $\mathcal{A}:\left[\beta_{1}, \alpha_{2}\right] \rightarrow E$ satisfying

$$
\beta_{1} \leq \mathcal{A} \beta_{1}, \mathcal{A} \alpha_{1} \leq \alpha_{1}, \beta_{2}<\mathcal{A} \beta_{2}, \mathcal{A} \alpha_{2} \leq \alpha_{2}
$$

has at least three fixed points, $u_{1}, u_{2}, u_{3}$ such that

$$
\beta_{1}<u_{1}<\alpha_{1}, \beta_{2}<u_{2} \leq \alpha_{2}, \beta_{2} \not \leq u_{3} \not \leq \alpha_{1} .
$$

Theorem 5. Assume that $\left(H_{1}\right)$ holds. Suppose that $\beta_{1}, \beta_{2}$ are LS, and $\alpha_{1}, \alpha_{2}$ are US of (1) such that $\beta_{2}, \alpha_{1}$ are strict with $\beta_{1}<\alpha_{1}<\beta_{2}<\alpha_{2}$. In this case, (1) has at least three solutions $u_{1}, u_{2}, u_{3}$ such that

$$
\beta_{1} \leq A \beta_{1}, \mathcal{A} \alpha_{1} \leq \alpha_{1}, \beta_{2}<\mathcal{A} \beta_{2}, \mathcal{A} \alpha_{2} \leq \alpha_{2}
$$

Proof. We shall show that $\mathcal{A}$ is strongly increasing operator. Equivalently saying, or all $u_{1}, u_{2} \in\left[\beta_{2}, \alpha_{2}\right]$ with $u_{1}<u_{2} u_{1}(y) \leq u_{2}(y)$ and $u_{1}(y) \neq u_{2}(y)$. In view of $\left(H_{1}\right)$, we have

$$
c\left(y, u_{1}\right)-c\left(y, u_{2}\right) \geq 0 \text { for all } y \in \Omega .
$$

As a result that there exists a neighborhood $\Omega^{\prime} \subset \Omega$ such that $u_{1}(y) \leq u_{2}(y)$ for $y \in \Omega^{\prime}$ since $u_{1}(y) \neq u_{2}(y)$. Hence, by $\left(H_{1}\right)$, we have for all $y \in \Omega$

$$
c\left(y, u_{1}\right)-c\left(y, u_{2}\right) \geq 0, y \in \Omega^{\prime} .
$$

By (8), we have

$$
\begin{aligned}
&-(\mathcal{A}-\sigma)\left(\mathcal{Q} u_{2}-\mathcal{Q} u_{1}\right)=c\left(y, u_{1}\right)-c\left(y, u_{2}\right)+\sigma\left(u_{2}-u_{1}\right) \\
& \geq-\sigma\left(u_{2}-u_{1}\right)+\sigma\left(u_{2}-u_{1}\right) \geq 0 \text { for all } y \in \Omega .
\end{aligned}
$$

Therefore, $\mathcal{Q} u_{2}<\mathcal{Q} u_{1}$ in $\Omega$ by strong maximum principle, and we conclude that $\mathcal{Q}$ is a strongly increasing operator.

Now, we prove $\beta_{1} \leq \mathcal{Q} \beta_{1}$. Consider the following problem:

$$
\left\{\begin{array}{lr}
-(\mathcal{A}-\sigma)\left(\mathcal{Q} \beta_{1}-\beta_{1}\right)=c\left(y, \beta_{1}\right)+\sigma \beta_{1}, & \text { in } \Omega, \\
\mathcal{N}\left(\mathcal{Q} \beta_{1}-\beta_{1}\right)=h, & \text { on } \partial \Omega .
\end{array}\right.
$$

In the view of $\beta_{1}$, an LS of (1), we have

$$
\begin{aligned}
-(\mathcal{A}-\sigma)\left(\mathcal{Q} \beta_{1}-\beta_{1}\right) & =-(\mathcal{A}-\sigma) \mathcal{Q} \beta_{1}+(\mathcal{A}-\sigma) \beta_{1} \\
& =-(\mathcal{A}-\sigma) \mathcal{Q} \beta_{1}+\mathcal{A} \beta_{1}-\sigma \beta_{1} \\
& =-(\mathcal{A}-\sigma) \mathcal{Q} \beta_{1}-\sigma \beta_{1}+\mathcal{A} \beta_{1} \\
& \geq c\left(y, \beta_{1}\right)-c\left(y, \beta_{1}\right)+\sigma \beta_{1}-\sigma \beta_{1} \geq 0 .
\end{aligned}
$$

Thus, $-(\mathcal{A}-\sigma)\left(\mathcal{Q} \beta_{1}-\beta_{1}\right) \geq 0$ and by strong maximum principle, we get $\mathcal{Q} \beta_{1} \geq \beta_{1}$.

In view of $\beta_{1}$, an LS of (1), we have

$$
\begin{aligned}
\mathcal{N}\left(\mathcal{Q} \beta_{1}-\beta_{1}\right) & =\mathcal{N}\left(\mathcal{Q} \beta_{1}\right)-\mathcal{N} \beta_{1} \\
& \geq \mathcal{B}\left(\mathcal{Q} \beta_{1}\right)-h \\
& =h-h=0,
\end{aligned}
$$


hence $\mathcal{N}\left(\mathcal{Q} \beta_{1}-\beta_{1}\right) \geq 0$, that is by strong maximum principle, we conclude $\mathcal{Q} \beta_{1} \geq \beta_{1}$. Then, $\mathcal{Q} \beta_{1} \geq \beta_{1}$.

Similarly, we have $\mathcal{Q} \beta_{2} \geq \beta_{2}$.

We know that $\mathcal{Q} \beta_{1} \neq \beta_{1}$. Since $\beta_{2}$ is an LS of (1), it is strict solution of (1). Thus, $\beta_{2}<T \beta_{2}$.

According to the same way, we can get

$$
\mathcal{A} \alpha_{1} \leq \alpha_{1}, \mathcal{A} \alpha_{2} \leq \alpha_{2}
$$

Thanks to the Theorem $4, \mathcal{Q}$ has at least three fixed points $u_{1}, u_{2}, u_{3}$ with

$$
\beta_{1}<u_{1}<\alpha_{1}, \beta_{2}<u_{2} \leq \alpha_{2}, \beta_{2} \not \leq u_{3} \not \alpha_{1} \text {. }
$$

Corollary 2. Assume that $\left(H_{1}\right)$ holds. Let $\beta_{1}, \beta_{2}$ be LSs and $\alpha_{1}$ be strict US of (1) such that $\beta_{1}<\alpha<\beta_{2}$. Then, (1) has at least three solutions $u_{1}, u_{2}, u_{3}$ such that

$$
\beta_{1} \leq \mathcal{A} \beta_{1}, \mathcal{A} \alpha_{1} \leq \alpha_{1}, \beta_{2}<\mathcal{A} \beta_{2}, \mathcal{A} \alpha_{2} \leq \alpha_{2}
$$

Proof. We shall apply Theorem 5. That is, assume that there are two upper solutions, $\alpha_{1}, \alpha_{2}$ satisfying:

$$
\beta_{1}(y) \leq \alpha_{1}(y)<\alpha_{2}(y)<\beta_{2}(y) \text { for } y \in \Omega \cup \partial \Theta,
$$

Let $\alpha_{1}$ and $\alpha_{2}$ be US such that $\alpha_{1}(y)<\alpha_{2}(y)$, for $y \in \Omega$ and

$$
\frac{\partial \alpha_{1}}{\partial v}(y)>\frac{\partial \alpha_{2}}{\partial v}(y), \text { for } y \in \partial \Theta
$$

Hence, we only verify that

$$
\alpha_{2}(y)<\beta_{2}(y) \text { for } y \in \Omega \text {. }
$$

Notice that

$$
\begin{gathered}
\beta_{2}(y)-\alpha_{1}(y)=0, \text { for } y \in \partial \Theta, \\
\beta_{2}(y)-\alpha_{1}(y)>0, \text { for } y \in \Omega \cup \partial \Theta .
\end{gathered}
$$

Provided that

$$
\frac{\partial \beta_{2}}{\partial v}(y)-\frac{\partial \alpha_{1}}{\partial v}(y)<0, \text { for } y \in \partial \Theta .
$$

Then, by strong maximum principle (Lemma 1), we have

$$
\beta_{2}(y)-\alpha_{1}(y)>0, \text { for } y \in \Omega,
$$

Suppose there is a $y_{0} \in \partial \Theta$ with

$$
\frac{\partial \beta_{2}}{\partial v}\left(y_{0}\right)-\frac{\partial \alpha}{\partial v}\left(y_{0}\right)=0 .
$$

Let $w=\beta_{2}-\alpha_{1}$. Since $\frac{\partial w}{\partial v}\left(y_{0}\right)>0$, we find an open ball $B_{y_{0}}$ such that $\frac{\partial w}{\partial v}(y)>0$, for all $y \in B_{y_{0}} \cap \bar{\Omega}$. Since $w(y)=0$ for $y \in \partial \Theta, w(y)<0$ for $y \in B_{y_{0}} \cap \Omega$, that implies:

$$
0<\beta_{2}(y)-\alpha_{1}(y)<0, \text { for } y \in B_{y_{0}} \cap \Omega,
$$

which is a contradiction. 


\section{Positive Global Solutions for Second Problem}

We are interested in existence of global solutions of (2). Suppose that $\mathcal{N}$ is defined either as

$$
\left\{\begin{array}{cr}
\mathcal{N} u(y, t)=u(y, t), & \text { on } \partial \Theta, \\
\mathcal{N} u(y, t)=\lambda u_{v}(y, t)+\mu u(y, t), & \text { on } \partial \Theta, \\
u(y, 0)=u_{0}(y) & \text { in } \Omega .
\end{array}\right.
$$

where the initial non-negative smooth map $u_{0}(y)$ satisfies compatibility condition $u_{0}(y)=0$ on $\partial \Omega$.

Recall that the operator $\mathcal{F}$ is defined as

$$
\mathcal{F} u=u_{t}-\mathcal{A} u \text {, where } \Delta u=\mathcal{A} u \text {. }
$$

As a matter of fact, we have that:

Definition 2. $\alpha(y, t) \in C(\bar{Q}) \cap C^{1,2}(\Theta)$ is a US of $(2)$ provided that

$$
\left\{\begin{array}{lr}
\mathcal{F} \alpha \geq c(y, t, \alpha), & \text { in } \Theta, \\
\mathcal{N} \alpha \geq h(y, t), & \text { on } \partial \Theta, \\
u(y, 0) \geq u_{0}(y), & \text { in } \Omega .
\end{array}\right.
$$

Similarly, $\beta(y, t) \in C(\bar{Q}) \cap C^{1,2}(\Theta)$ is an LS by changing direction of inequalities in (9), we set $u \in C(\bar{\Theta})$ with $\beta \leq u \leq \alpha$ in $\bar{\Theta}$.

It is obvious that the upper and lower solutions of (2) are given by $\alpha(y, t), \beta(y, t)$. Let $\sigma$ be at $\left(H_{1}\right)$ with

$$
c\left(y, u_{1}\right)-c\left(y, u_{2}\right)+\sigma\left(u_{1}-u_{2}\right)>0,
$$

on

$$
\min \beta(y, t) \leq u_{i} \leq \max \alpha(y, t), i=1,2
$$

We define $\bar{u}^{(1)}$ as

$$
\left\{\begin{array}{lr}
\mathcal{F} \bar{u}^{(1)}+\sigma \bar{u}^{(1)}=c(y, t, \alpha)+\sigma \alpha & \text { in } \Theta, \\
\mathcal{N} \bar{u}^{(1)}=h, & \text { on } \partial \Theta, \\
u^{(1)}(y, 0)=u_{0}(y), & \text { in } \Omega .
\end{array}\right.
$$

Then, by the maximum principle for a parabolic equation, we have

$$
\bar{u}^{(1)}(y, t)<\alpha(y, t), \text { in } \Omega .
$$

Defining $\psi: \alpha(y, t) \mapsto \bar{u}^{(1)}(y, t)$, we have $\bar{u}^{(1)}=\psi \alpha$ is a monotone operator with type of Collatz [30]. Letting $\underline{u}^{(1)}=\psi \beta$, we get

$$
\left\{\bar{u}^{(n)}\right\},\left\{\underline{u}^{(n)}\right\},
$$

with

$$
\bar{u}^{(n)}=\psi \bar{u}^{(n-1)},
$$

and

$$
\underline{u}^{(n)}=\psi \underline{u}^{(n-1)},
$$


in which

$$
\bar{u}^{(1)}=\psi \alpha, \underline{u}^{(1)}=\psi \beta .
$$

Theorem 6. Suppose that conditions of $\left(H_{1}\right)$ hold. Assume also that $\alpha(y, t) \in \bar{\Theta}, \beta(y, t) \in \bar{\Theta}$ are a US and LS of (2). If there is a $\sigma$ such that

$$
c\left(y, u_{1}\right)-c\left(y, u_{2}\right)+\sigma\left(u_{1}-u_{2}\right)>0
$$

where

$$
\min _{\Omega} \beta \leq u_{i} \leq \max _{\Omega} \alpha, i=1,2,
$$

there exists a unique strong solution $u$ of (2) with

$$
\lim _{n \rightarrow \infty} \bar{u}^{(n)}=\psi u=u=\lim _{n \rightarrow \infty} \underline{u}^{(n)},
$$

where $\left\{\bar{u}^{(n)}\right\}$ is decreasing and $\left\{\underline{u}^{(n)}\right\}$ is increasing sequences. We address the situation where $h$ is time independent next.

Corollary 3. Suppose that conditions of $\left(H_{1}\right)$ hold. If $u(y) \in(\underline{u}(y), \bar{u}(y))$ is a solution of

$$
\begin{cases}-\mathcal{A} u=c(y, u(y)), & \text { in } \Omega, \\ \mathcal{N} u=h, & \text { on } \partial \Omega,\end{cases}
$$

where $\bar{u}(y)$ and $\underline{u}(y)$ are an upper and an LS, respectively, there is a global regular solution $u(y, t) \in$ $(\underline{u}(y), \bar{u}(y))$, for all $t>0$.

Now, we introduce two identities:

$$
\begin{cases}-\mathcal{A} u=c(y, u), & \text { on } \Theta, \\ u=0, & \text { on } \partial \Omega,\end{cases}
$$

and

$$
\left\{\begin{array}{l}
\mathcal{F} u=c(y, t, u), \quad \text { in } \Theta, \\
u=0, \quad \text { on } \partial \Theta \\
u(y, 0)=\bar{u}(y), \quad \text { in } \Omega .
\end{array}\right.
$$

Theorem 7. Suppose that conditions of $\left(H_{1}\right)$ hold and also $\bar{u}(y)$ is a US of $(10)$. If $u(y, t)$ is a solution of $(11)$, $u_{t} \leq 0$.

Proof. Assume $\left.\left\{u^{(} n\right)(y, t)\right\} \subset \Theta$ is a sequence of maps by $u^{(0)}(y, t)=\bar{u}(y)$, and for $n \geq 1$,

$$
\left\{\begin{array}{lr}
\mathcal{F} u^{(n)}+\sigma u^{(n)}=c\left(y, u^{(n-1)}\right)+\sigma u^{(n-1)}, & \text { in } \Theta, \\
u^{(n)}=0, & \text { on } \partial \Theta \\
u^{(n)}(y, 0)=\bar{u}(y), & \text { in } \Omega .
\end{array}\right.
$$

In this case,

$$
\bar{u}(y) \geq u^{(1)}(y, t) \geq \ldots \geq u^{(n-1)}(y, t) \geq u^{(n)}(y, t) \geq \ldots
$$


Recall that

$$
\left\{\begin{array}{l}
\mathcal{F}\left(u^{(1)}-\bar{u}\right)+\sigma\left(u^{(1)}-\bar{u}\right)=-[c(y, \bar{u})-\mathcal{A} \bar{u}] \geq 0 \\
\mathcal{N}\left(u^{(1)}-\bar{u}\right)=h(y, t)-\mathcal{N} \bar{u} \leq 0
\end{array}\right.
$$

We conclude by strong maximum principle that $\bar{u} \geq u^{(1)}$.

By induction and from (13) for $n \in \mathbb{N}$, we deduce the existence

$$
u^{(n)} \rightarrow u^{*} \text { as } n \rightarrow \infty .
$$

Thus, $u^{*}(y, t)$ is a solution of

$$
\left\{\begin{array}{lr}
\mathcal{F} u^{*}=c\left(x, u^{*}\right), & \text { in } \Theta, \\
u^{*}=0, & \text { on } \partial \Theta, \\
u^{*}(y, 0)=\bar{u}(y), & \text { in } \Omega,
\end{array}\right.
$$

Hence, $u^{*}(y, t)=u(y, t) \in \Theta$, via uniqueness condition. Differentiating (12) with respect to $t$, we have

$$
\begin{cases}\mathcal{F}\left(u^{(n)}\right)_{t}+\sigma\left(u^{(n)}\right)_{t}=c_{U}(y, U) U_{t}, & \text { in } \Theta, \\ \left(u^{(n)}\right)_{t}=0, & \text { on } \partial \Theta\end{cases}
$$

in which $U=u^{(n-1)}$.

Since $c_{U}(y, U) U_{t}$ is located in $\Theta$, it is bounded.

We set

$$
w_{n}=\frac{u^{(n)}(y, \delta)-u^{(n)}(y, 0)}{\delta}, y \in \Omega . \delta>0,
$$

As a result by (12) and (13), we get $w_{n} \leq 0$. Hence, $\left(u^{(n)}(y, 0)\right)_{t} \leq 0, y \in \Omega$. Furthermore, we have $\left(u^{(n)}\right)_{t} \leq 0$.

We can apply the same proof of Theorem 1 to get

$$
u^{(n)} \mapsto u \in C^{1+\eta} \text { on } t \text {, for } 0<\eta<1 .
$$

Thus, $u_{t}(y, t) \leq 0$ in $\Theta$. Herewith, the proof is complete.

Let us assume $c(y, t, u)$ is a $C^{1}$-mapping for $u$ and satisfies the inequalities:

$$
\begin{cases}c\left(y, t, c_{1}\right) \geq 0, \quad c\left(y, t, c_{2}\right) \leq 0, & \text { in } \Omega \cap \mathbb{R}^{+}, \\ c_{1} \mu(y, t) \leq h(y, t) \leq c_{2} \mu(y, t), & \text { on } \partial \Omega \cap \mathbb{R}^{+},\end{cases}
$$

where $c_{1}>0, c_{2}>0$ are constants with $c_{1}<c_{2}$.

Theorem 8. Assume that (14) holds. If there exists constants $c_{1}>0, c_{2}>0$ with $c_{1}<c_{2}$. Then, for all $u \in\left[c_{1}, c_{2}\right],(2)$ has a unique positive and bounded global solution.

Proof. Let $\alpha=c_{2}, \beta=c_{1}$; then, by (14), we get

$$
\left\{\begin{array}{lr}
\mathcal{F} \alpha=0 \geq c\left(y, t, c_{2}\right)=c(y, t, \alpha), & \text { in } \Omega \times \mathbb{R}^{+}, \\
\mathcal{N} \alpha=\lambda \alpha_{v}+\mu \alpha=c_{2} \mu(y, t) \geq h(y, t), & \text { on } \partial \Omega \times \mathbb{R}^{+}, \\
\alpha=c_{2}, & \text { in } \Omega .
\end{array}\right.
$$


This allows to conclude that $\alpha=c_{2}$, is a US. Similarly, $\beta=c_{1}$ is an LS. Consequently, thanks to the Theorem 6, we conclude the result.

We are now ready to prove the uniqueness result of global positive solution. To this purpose, we assume that:

(a) For every $y \in \Omega, y^{\prime} \in \partial \Omega: c(y, t) \geq 0, h\left(y^{\prime}, t\right) \geq 0$ and $u_{0}(y) \geq 0$.

Theorem 9. Suppose that (a), (5) and (6) hold. If there exists a mapping $M$ with

$$
c_{u}(y, t, u) u \leq M(y, t) u, \text { for every } T<\infty, u \geq 0, \text { in } \Theta,
$$

then, (2) has a unique global positive solution.

Proof. Using the mean-value theorem, (5) and (6), we have

$$
\begin{aligned}
c(y, t, u) & =c_{u}(y, t, \xi) u+c(y, t, 0), \\
& \geq c_{u}(y, t, \xi) u, \text { in } \Theta,
\end{aligned}
$$

in which $\xi=\xi(y, t)$ is intermediate value between $u$ and 0 .

By Lemma 3 and $\mathcal{F}$, we write

$$
c_{3}(y, t)=-c_{u}(y, t, \xi) .
$$

Hence, $u=0$, or $0<u$ in $\Theta . u$ is positive because, if it is not, $u=0$ only if all of the maps in $(a)$ is equal to 0 . This implies that $u=\beta$ is an LS of (2).

Let $w$ be a solution of:

$$
\left\{\begin{array}{lr}
\mathcal{F} w=M w+c(y, t, 0) & \text { in } \Theta, \\
\mathcal{N} w=h(y, t) & \text { on } \partial \Theta \\
w(y, 0)=u_{0}(y) & \text { in } \Omega
\end{array}\right.
$$

Therefore, $w$ is a upper positive solution of (2). As a matter of fact, for $\alpha=w$ and again applying the mean-value theorem,

$$
c(y, t, \alpha)=c_{\alpha}(y, t, \xi)(\alpha)+c(y, t, 0),
$$

where $\xi=\xi(y, t)$ is located between $\alpha$ and 0 .

Combining with $(a),(5),(6)$ and (15), we have

$$
c(y, t, \alpha) \leq M(y, t) \alpha+c(y, t, 0), \text { in } \Theta .
$$

Thus,

$$
\begin{cases}\mathcal{F} \alpha=M \alpha+c(y, t, 0) \geq c(y, t, \alpha), & \text { in } \Theta, \\ \mathcal{N} w=h(y, t) \geq 0, & \text { on } \partial \Theta, \\ w(y, 0)=u_{0}(y) \geq 0, & \text { in } \Omega,\end{cases}
$$

which implies that $\alpha$ is an upper positive solution of (2). Hence, by Theorem 8 , we deduce the unique positive global solution of (2).

\section{Weak Solutions for the First Problem}

Now, our main result shows the existence of a weak solution to problem (1) with Dirichlet boundary condition $(u=0$ i.e., $h=0$ and $\mathcal{N} u=u)$ under a unboundedness on $c$. Before doing this, we introduce the following notion of weak solution to (1). For that, we need Lemmas 5-7 and the assumptions $\left(H_{3}\right)$ and $\left(H_{4}\right)$ (see later). Note that the notion of weak solution to $(1)$ is essentially the same as in Definition 1, the only difference is that we now require that $u$ belong to $H_{0}^{1}(\Omega)$. 
Definition 3. Let $u \in H_{0}^{1}(\Omega)$, $u$ is said to be a weak solution of (1) if it satisfies

$$
\int_{\Omega} \nabla u \nabla \phi d x=\int_{\Omega} c(u) \phi d x \text { in } \Omega .
$$

A nonnegative function $\underline{u}, \bar{u} \in H_{0}^{1}(\Omega)$ is called a weak lower solution (WLS) and weak upper solution (WUS) of (1) if they satisfy

$$
\int_{\Omega} \nabla \underline{u} \nabla \phi d x \leq \int_{\Omega} c(\underline{u}) \phi d x \text { in } \Omega,
$$

and

$$
\int_{\Omega} \nabla \bar{u} \nabla \phi d x \geq \int_{\Omega} c(\bar{u}) \phi d x \text { in } \Omega
$$

for all $\phi \in H_{0}^{1}(\Omega)$.

Lemma 5. ([26]) Let $v$ solve $\Delta v=c$ in $\Omega$. If $c \in C(\Omega)$, then $v \in C^{1, \eta}(\Omega)$ for any $\eta \in(0,1)$, thus in particular, $v$ is continuous in $\Omega$.

Lemma 6. ([32]) For each $c \in L^{2}(\Omega)$. Then, there exists a unique solution $v \in H_{0}^{1}(\Omega)$ to problem (1).

Lemma 7. ([33]) Assume that $u$ and $v$ are two non-negative functions such that

$$
\left\{\begin{array}{l}
-\Delta u \geq-\Delta v, \quad \text { in } \Omega, \\
u=v=0, \quad \text { in } \partial \Omega .
\end{array}\right.
$$

Then, $u \geq v$, a.e., in $\Omega$.

$\left(H_{3}\right) c \in C^{1}((0,+\infty))$ is increasing function such that

$$
\lim _{u \rightarrow+\infty} c(u)=+\infty .
$$

$\left(H_{4}\right)$ Moreover, $c \in C^{1}((0,+\infty))$ satisfies

$$
\lim _{u \rightarrow+\infty} \frac{c(u)}{u}=0
$$

Theorem 10. Let $\left(H_{3}\right)$ and $\left(H_{4}\right)$ hold. Then, problem (1) has a positive weak solution.

Proof. Let $\sigma$ be the first eigenvalue of $-\Delta$ with Dirichlet boundary conditions and $\phi_{1}$ be the corresponding positive eigenfunction with $\left\|\phi_{1}\right\|=1$.

Let $\delta>0$ be such that $\left|\nabla \phi_{1}\right|^{2}-\sigma \phi_{1}^{2}>0$ on

$$
\bar{\Omega}_{\delta}=\{x \in \Omega: d(x, \partial \Omega) \leq \delta\} .
$$

Let us define

$$
\underline{u}=\frac{1}{2} \phi_{1}^{2} .
$$

We shall verify that $\underline{u}$ is a weak LS of (1). Indeed, let $\phi \in H_{0}^{1}(\Omega)$ with $\phi>0$ in $\Omega$. A simple calculation shows that

$$
\int_{\bar{\Omega}_{\delta}} \nabla \underline{u} \nabla \phi d x=\int_{\bar{\Omega}_{\delta}} \phi_{1} \nabla \phi_{1} \nabla \phi d x
$$




$$
\begin{aligned}
& =\int_{\bar{\Omega}_{\delta}} \nabla \phi_{1} \nabla\left(\phi_{1} \cdot \phi\right) d x-\int_{\bar{\Omega}_{\delta}}|\nabla \phi|^{2} \phi d x \\
& =\int_{\bar{\Omega}_{\delta}}\left(\sigma \phi_{1}^{2}-|\nabla \phi|^{2}\right) \phi d x .
\end{aligned}
$$

On $\bar{\Omega}_{\delta}$, we have $\left|\nabla \phi_{1}\right|^{2}-\sigma \phi_{1}^{2}>0$, then $\sigma \phi_{1}^{2}-\left|\nabla \phi_{1}\right|^{2}<0$. Hence,

$$
\int_{\bar{\Omega}_{\delta}} \nabla \underline{u} \nabla \phi d x<0 .
$$

By $\left(H_{3}\right)$, we get $c(\underline{u})>0$ (large enough).

Then,

$$
\int_{\bar{\Omega}_{\delta}} \nabla \underline{u} \nabla \phi d x \leq \int_{\bar{\Omega}_{\delta}} c(\underline{u}) \phi d x .
$$

Next, on $\Omega \backslash \bar{\Omega}_{\delta}$, we have $\phi_{1} \geq d$ for some $d>0$. By $\left(H_{3}\right)$ and by the definition of $\underline{u}$, it follows that:

$$
\begin{aligned}
\int_{\Omega \backslash \bar{\Omega}_{\delta}} c(\underline{u}) \phi d x & \geq \int_{\Omega \backslash \bar{\Omega}_{\delta}} \sigma \phi d x \\
& \geq \int_{\Omega \backslash \bar{\Omega}_{\delta}}\left(\sigma \phi_{1}^{2}-|\nabla \phi|^{2}\right) \phi d x \\
& =\int_{\bar{\Omega}_{\delta}} \nabla \underline{u} \nabla \phi d x,
\end{aligned}
$$

From (17) and (18), we deduce that

$$
\int_{\Omega} \nabla \underline{u} \nabla \phi d x \leq \int_{\Omega} c(\underline{u}) \phi d x,
$$

for any $\phi \in H_{0}^{1}(\Omega)$. That is, $\underline{u}$ is a weak LS of problem (1).

Next, we shall construct a WUS of (1). Let $e$ be the solution of the following problem

$$
\begin{cases}\Delta e=1 & \text { in } \Omega, \\ e=0, & \text { on } \partial \Omega .\end{cases}
$$

Let $\bar{u}=C e$, where $C$ is a positive real number which will be chosen later. We will verify that $\bar{u}$ is a weak US (1). Let $\phi \in H_{0}^{1}(\Omega)$ with $\phi>0$ in $\Omega$. Then, from (19), we get

$$
\begin{aligned}
\int_{\Omega} \nabla \bar{u} \nabla \phi d x & =C \int_{\Omega} \nabla e \nabla \phi d x \\
& =C \int_{\Omega} \phi d x .
\end{aligned}
$$

By $\left(H_{3}\right)$, we can choose $C$ large enough so that

$$
C \geq c\left(C\|e\|_{\infty}\right) .
$$


Therefore,

$$
\begin{aligned}
\int_{\Omega} \nabla \bar{u} \nabla \phi d x & \geq c\left(C\|e\|_{\infty}\right) \int_{\Omega} \phi d x \\
& \geq \int_{\Omega} c\left(C\|e\|_{\infty}\right) \phi d x \\
& \geq \int_{\Omega} c(\bar{u}) \phi d x .
\end{aligned}
$$

that is, $\bar{u}$ a weak US of (1) for a large enough $C$. In order to obtain a weak solution of (1), we define the sequence

$$
\left\{u_{n}\right\} \subset E=H_{0}^{1}(\Omega) \cap C(\Omega),
$$

as $u_{0}=\bar{u} \subset E=H_{0}^{1}(\Omega) \cap C(\Omega)$, and $u_{n}$ is the unique solution of the problem

$$
\begin{cases}-\Delta u_{n}=c\left(u_{n-1}\right) & \text { in } \Omega, \\ u=0, & \text { in } \partial \Omega .\end{cases}
$$

If $u_{n-1} \in E$ is given, the right-hand-side of $(20)$ is independent of $u_{n}$ since $c\left(u_{0}\right) \in C(\Omega) \subset L^{2}(\Omega)$ in $x$ and from Lemma 6 . Then, (20) with $n=1$ has unique solution $u_{1} \in H_{0}^{1}(\Omega)$.

We deduce from Lemma 5 that $u_{1} \in C(\Omega)$. Consequently, we conclude that $u_{1} \in E$. In the same way, we construct the following elements $u_{n} \in E$ of our sequence. From (20), and by the fact that $u_{0}$ is a weak US of (1), we have

$$
\left\{\begin{array}{lr}
-\Delta u_{0} \geq c\left(u_{0}\right)=-\Delta u_{1}, & \text { in } \Omega, \\
u=0, & \text { in } \partial \Omega,
\end{array}\right.
$$

from which and Lemma 7 , we have $u_{0} \geq u_{1}$.

Since $u_{0} \geq \underline{u}$ and by the monotonicity of $c$, we have

$$
-\Delta u_{1}=c\left(u_{0}\right) \geq c(\underline{u}) \geq-\Delta \underline{u} .
$$

from which and Lemma 7, we deduce that $u_{1} \geq \underline{u}$.

From (20), and by the monotonicity of $c, u_{1} \geq \underline{u}$ and $u_{0} \geq u_{1}$, for $u_{2}$, we write

$$
-\Delta u_{1}=c\left(u_{0}\right) \geq c\left(u_{1}\right)=-\Delta u_{2},
$$

and

$$
-\Delta u_{2}=c\left(u_{1}\right) \geq c(\underline{u}) \geq-\Delta \underline{u} .
$$

Then, thanks to the Lemma 7 , we get $u_{1} \geq u_{2}$ and $u_{2} \geq \underline{u}$. Repeating this argument, we get a bounded monotonic sequence $\left\{u_{n}\right\}$ satisfying

$$
\bar{u}=u_{0} \geq u_{1} \geq \cdots \geq u_{n} \geq \underline{u}>0 .
$$

Thanks to the continuity of the function $c$ and by the definition of the sequences $\left\{u_{n}\right\}$, there exists a constant $\mathcal{R}$, which is independent from $n$ such that

$$
\left|c\left(u_{n-1}\right)\right|<\mathcal{R} .
$$

Using (21), multiplying the first equation of (20) by $u_{n}$, integrating and using the Hölder inequality and Sobolev's embedding, we can show that 


$$
\begin{aligned}
\int_{\Omega}\left|\nabla u_{n}\right|^{2} d x & \leq \int_{\Omega} c\left(u_{n}\right) u_{n} d x \\
& \leq \mathcal{R} \int_{\Omega}\left|u_{n}\right| d x \\
& \leq \mathcal{R}\left\|u_{n}\right\|_{H_{0}^{1}(\Omega)} .
\end{aligned}
$$

Then,

$$
\left\|u_{n}\right\|_{H_{0}^{1}(\Omega)} \leq \mathcal{R}, \quad \text { for. all } n .
$$

where $\mathcal{R}^{\prime}$ is a constant and independent from $n$. By (22), we infer that $\left\{u_{n}\right\}$ has a subsequence which weakly converges in $H_{0}^{1}(\Omega, \mathbb{R})$ to $u$ with $u \geq \underline{u}>0$. Now, letting $n \rightarrow+\infty$, we deduce that $u$ is a positive weak solution of (1). Hereby, the proof is completed.

\section{Conclusions and Outlook}

There have been two main objectives in this paper. As explained in introduction part, the first one is from the lack of existence of multiple solutions by using the bifurcation theory. Second one is concerning multiplicity of more than two solutions. We presented new original theorems regarding the existence of positive solutions of nonlinear elliptic BVPs (Theorem 10). Extension of obtained results to fractional-stochastic PDEs will be investigated in a future research work.

Author Contributions: All of conceptualization, methodology, validation, M.I., N.B., M.A.A., S.B., Y.-M.C., G.-W.W. and B.A. All authors have read and agreed to the published version of the manuscript.

Funding: The work was supported by the Natural Science Foundation of China (Grant Nos. 61673169, 11301127, 11701176, 11626101, 11601485). B. Almohsen is supported by Researchers Supporting Project number (RSP-2020/158), King Saud University, Riyadh, Saudi Arabia.

Conflicts of Interest: The authors declare that there is no conflict of interests regarding the publication of this paper.

\section{Abbreviations}

The following abbreviations are used in this manuscript:

BVP Boundary Value Problems

NEBVP Nonlinear Elliptic Boundary Value Problems

PDEs Partial Differential Equations

US Upper Solution

LS Lower Solution

SU Strictly Upper

SL Strictly Lower

WLS Weak lower Solution

WUS Weak upper Solution

\section{References}

1. Chipot, M.; Lovat, B. On the asymptotic behaviour of some nonlocal problems. Positivity 1999, 3, 65-81. [CrossRef]

2. Ako, K. On the Dirichlet problem for quasi-linear elliptic differential equations of second order. J. Math. Soc. Jpn. 1961, 13, 45-62. [CrossRef]

3. Amann, H.; Laestch, T. On the existence of positive solutions of nonlinear elliptic BVPs. Indiana Univ. Math. J. 1971, 13, 125-146. [CrossRef]

4. Afrouzi, G.A.; Naghizadeh, Z.; Mahdavi, S. Monotone methods in nonlinear elliptic BVP. Int. J. Nonlinear Sci. 2009, 7, 283-289.

5. Bouteraa, N.; Benaicha, S. Existence of solutions for third-order three-point BVP. Mathematica 2018, 60, 12-22. 
6. Bouteraa, N.; Benaicha, S. Existence and multiplicity of positive radial solutions to the Dirichlet problem for nonlinear elliptic equations on annular domains. Stud. Univ. Babes-Bolyai Math. 2020, 65, 109-125. [CrossRef]

7. Bouteraa, N.; Benaicha, S.; Djourdem, H. On the existence and multiplicity of positive solutions for nonlinear elliptic equation on bounded annular domains via fixed point index. Maltepe J. Math. 2019, I, 30-47.

8. Cano-Casanova, C. Linear elliptic and parabolic PDEs with nonlinear mixed boundary conditions and spacial heterogeneties. Electron. J. Differ. Equ. 2018, 166, 1-27.

9. Keller, H.K. Elliptic BVPs suggested by nonlinear diffusion processes. Arch. Rat. Mech. Anal. 1969, 5, 363-381. [CrossRef]

10. Ma, R.; Chen, R.; Lu, Y. Method of Lower and Upper Solutions for Elliptic Systems with Nonlinear Boundary Condition and Its Applications. J. Appl. Math. 2014, 2014, 705298. [CrossRef]

11. Nagumo, M. On principally linear elliptic differential equations of second order. Osaka Math. J. 1954, 6, 207-229.

12. Puel, J.P. Existence comportement a l'infini et stabilite dans certaines problemes quasilineares elliptiques et paraboliques d'ordre 2. Ann. Scuola Norm. Sup. Pisa 1977, IV 3, 89-119.

13. Pao, C.V. Asymptotic behavior and nonexistence of global Solutions for a class of nonlinear BVPs of Parabolic Type. J. Math. Anal. Appl. 1978, 65, 616-637. [CrossRef]

14. Pao, C.V.; Ruan, W.H. Positive solutions of quasilinear parabolic systems with Dirichlet boundary condition. J. Math. Anal. Appl. 2007, 333, 472-499. [CrossRef]

15. Sattinger, D.H. Monotone methods in nonlinear elliptic and parabolic BVPs. Ind. J. Math. 1972, 211, $979-1000$. [CrossRef]

16. Kropat, E.; Pickl, S.; Rößler, A.; Weber, G.-W. A new algorithm from semi-infinite optimization for a problem of time-minimal control. J. Comput. Technol. 2000, 5, 67-81.

17. Yilmaz, F.; Öz, H.; Weber, G.-W. Simulation of Stochastic Optimal Control Problems with Symplectic Partitioned Runge-Kutta Scheme. Dyn. Contin. Discret. Impuls. Syst. Ser. B 2015, 22, 425-440.

18. Öz, H.; Yilmaz, F.; Weber, G.-W. A discrete optimality system for an optimal harvesting problem. Comput. Manag. Sci. 2017, 14, 519-533.

19. Öz Bakan, H.; Yilmaz, F.; Weber, G.-W. Minimal Truncation Error Constants for Symplectic Partitioned Runge-Kutta Method for Stochastic Optimal Control Problems. J. Comput. Appl. Math. (JCAM) 2018, 331, 196-207. [CrossRef]

20. Yilmaz, F.; Öz, H.; Weber, G.-W. Calculus and Digitalization in Finance: Change of Time Method and Stochastic Taylor Expansion with Computation of Expectation. In Modeling, Optimization, Dynamics and Bioeconomy I; Springer: Berlin, Germany, 2014; Volume 73, pp. 739-753, Chapter 40.

21. Kim, M.-K. Optimal Control and Operation Strategy for Wind Turbines Contributing to Grid Primary Frequency Regulation. Appl. Sci. 2017, 7, 927. [CrossRef]

22. Yang, D.; Zhao, K.; Tian, H.; Liu, Y. Decision Optimization for Power Grid Operating Conditions with Highand Low-Voltage Parallel Loops. Appl. Sci. 2017, 7, 487. [CrossRef]

23. Sánchez, A.J.F. Existence and Multiplicity of Solutions for Elliptic Problems with Critical Growth in the Gradient. 2019; pp. 1-167. Available online: https://tel.archives-ouvert.fr/tel-02299049 (accessed on 7 July 2020).

24. Ovono, A.A.; Rougirel, A. Elliptic equations with diffusion parameterized by the range of nonlocal interactions. Ann. Mat. Pura Appl. 2010, 1, 163-183. [CrossRef]

25. Alves, C.O.; Covei, D.P. Existence of solution for a class of nonlocal elliptic problem via sub-supersolution method. Nonlinear Anal. Real Word Appl. 2015, 23, 1-8. [CrossRef]

26. Diaz, J.I.; Goomez-Castro, D. An application of shape differentiation to the effectiveness of steady state reaction-diffusion problem arising in chemical engineering. Electron. J. Differ. Equ. 2015, 2, 31-45.

27. Yan, B.; Ren, Q. Existence, uniqueness and multiplicity of positive solutions for some nonlocal singular elliptic problems. Electron. J. Differ. Equ. 2017, 138, 1-21.

28. Yan, B.; O'Regan, D.; Agarwal, R.P. The existence of positive solutions for Kirchhoff-type problems via the sub-supersolution method. An. St. Univ. Ovidius Constanta 2018, 26, 5-41. [CrossRef]

29. Troianiello, G.M. Elliptic Differential Equations and Obstacle Problems; Plenum: New York, NY, USA, 1987.

30. Collatz, L. Functional Analysis and Numerical Mathematics; Academic Press Inc.: New York, NY, USA; London, UK, 1966. 
31. Amann, H. Fixed point equations and nonlinear eigenvalue problems in ordered Banach spaces. SIAM Rev. 1976, 18, 620-709. [CrossRef]

32. Alves, C.O.; Correa, F.G.S.A. On existence of solutions for a class of problem involving a nonlinear operator. Comm. Appl. Nonlinear Anal. 2001, 8, 43-56.

33. Azouz, N.; Bensedik, A. Existence result for an elliptic equation of Kirchhoff type with changing sign data. Funkcial. Ekvac. 2012, 55, 55-66. [CrossRef]

(C) 2020 by the authors. Licensee MDPI, Basel, Switzerland. This article is an open access article distributed under the terms and conditions of the Creative Commons Attribution (CC BY) license (http://creativecommons.org/licenses/by/4.0/). 